\title{
THE PARTITIONING METHOD BASED ON HEDGE ALGEBRAS FOR FUZZY TIME SERIES FORECASTING
}

\author{
Hoang Tung ${ }^{1, *}$, Nguyen Dinh Thuan', Vu Minh Loc ${ }^{2}$ \\ ${ }^{1}$ University of Information Technology, Linh Trung Ward, Thu Duc Dist, Ho Chi Minh City \\ ${ }^{2}$ Ba Ria-Vung Tau University, 80 Truong Cong Dinh Str, Vung Tau City, Ba Ria-Vung Tau Pro \\ *Email: tung_k51e@yahoo.com
}

Received: 27 October 2015; Accepted for publication: 19 June 2016

\begin{abstract}
In recent years, many partitioning methods have been proposed for fuzzy time series, because they strongly affect to forecasting results. In this paper, we present a novel partitioning method based on hedge algebras (HA). The experimental results show that the proposed method is better than the others on the accuracy of forecasting. It is simple and flexible in applying this method because we can determine the parameters of HA for reasonable intervals.
\end{abstract}

Keywords: fuzzy time series, forecasting time series, reasonable intervals, hedge algebras.

\section{INTRODUCTION}

In the first research on the fuzzy time series in 1993, Song and Chissom [1] proposed a method (S\&C) that used fuzzy time series to forecast time series. According to that, $C(t)$ is the conventional time series that needs to be forecasted, this one can be forecasted by converting into fuzzy time series $F(t)$. After that, the forecasting result on $F(t)$ is defuzzified to become the forecasting result on $C(t)$. So, $F(t)$ is a qualitative view about $C(t)$. Because of this, we offer a convention by giving the collection of all historical values of $F(t)$ to be $C(t)$ and the values of $F(t)$ to be the linguistic terms that are used to qualitatively describe the values of $C(t)$. The method S\&C can be summarized in seven steps: (1) Determining $U$ which is the universe of discourse of $F(t)$, (2) Partitioning $U$ into a collection of intervals, (3) Determining the collection of linguistic terms used to quanlitatively describe the historical values of $F(t)$, (4) Quantifying linguistic terms by means of fuzzy sets, (5) Mining fuzzy relationships, $A_{n} \rightarrow A_{m^{\circ}} R_{i}$ where $i=1,2$, $\ldots, A_{n}, A_{m}$ and $R_{i}$ respectively are fuzzy sets used to quantify the values of $F(t)$ at point $t-1, t$ and fuzzy relation between these values, (6) Calculating forecasting values by the formula $A_{a}=A_{b^{\circ}} R$ (*), in which $A_{a}$ and $A_{b}$, the values of $F(t)$, are quantified by fuzzy sets at point $t, t-1$, and $R=$ $\cup R_{i}$; (7) Defuzzifying forecasting values on $F(t)$ to find forecasting values of $C(t)$. Song and Chissom, in [2,3], used S\&C to forecast enrollments at University of Alabama. 
We can see that step (2), in the method of Song and Chissom, plays a pivotal role because this step significatly impacts remaining steps and forecasting accuracy. Indeed, if we increase the number of intervals, then we have to get larger computation overhead for performing steps (6), (7) and these steps directly affect to forecasting result. So, how to partition the universe of discourse (how to partition $U$ ) has become a basic problem in the field of using fuzzy time series to forecast time series.

In 1996, Chen proposed an improved method for using fuzzy time series to forecast enrollments at University of Alabama [4]. This research is remarkable because one used simple arithmetic operations on intervals to compute forecasting values and to significantly reduce calculation time. The most impressive thing is that it has spread a new idea in studying fuzzy time series, in which, researchers just focus on finding reasonable intervals.

Up to now, based on the studies, we can distinguish between two types of partitioning $U$ : partitioning $U$ into equal or not equal intervals. The studies in [2 - 8] are typical for the first type. The papers [9-15] are delegated for the second type. Generally, the studies follow the second type of partitioning that are newer ones and usually yield better forecasting result than the others. There are rather many ways to partition $U$ following second type. For instance, in [9] Chen et al. based on statistical distribution of historical values in each interval, in [10] Huarng et al. based on ratio between two consecutive historical values, Chen and Kao in [11] employed particle swarm optimization, Wang et al. in [12, 13] used information granules, Bas in [14] exploited modified genetic algorithm, and $\mathrm{Lu}$ et al. in [15] also used information granules to partition $U$.

As already mentioned, the second type of partitioning gives more accuracy forecasting result than the others, but, it is quite difficult to find intervals following the second type based on the approaches same as [9-15]. At the same time, the quality of forecasting result is not good enough. In this paper, we present a novel method of partitioning the universe of discourse based on hedge algebras (HA). We can get reasonable intervals with the proposed method.

According to this method, the number of intervals, partitioned on $U$, are equal to the number of linguistic terms used to qualitatively describe the historical values of fuzzy time series and fuzziness interval of each linguistic term is assigned to size of each interval. As a result, intervals can have not equal size. The experimental results show that proposed method has better forecasting performance, on regular time series, than the others published recently.

The rest of this paper is organized as follows. In Section 2, we briefly introduce some basic concepts in HA. The main content of the paper, novel method of partitioning the universe of discourse based on HA, is presented in Section 3. Section 4 presents experimental result and some discussions for applying the proposed method to forecast on some regular time series. Section 5, the last section, is the conclusion of the paper.

\section{SOME BASIC CONCEPTS IN HEDGE ALGEBRAS}

In this section, we refer to paper $[16,17]$ to briefly review some basic concepts in HA, these concepts are exploited to form the proposed method.

The HA are denoted by $A X=(X, G, C, H, \leq)$, where, $G=\left\{c^{+}, c^{-}\right\}$is the collection of primary generators, in which $c^{+}$and $c^{-}$are, respectively, the negative primary term and the positive one of a linguistic variable $X, C=\{0,1, W\}$ is a set of constants, which are distinguished with elements in $X, H$ is the set of hedges, " $\leq$ " is a semantically ordering relation on $X$. For each $x \in X$ in HA, $H(x)$ is the set of terms $u \in X$ generated from $x$ by applying the 
hedges of $H$ and $u=h_{n} \ldots h_{1} x$, with $h_{n}, \ldots, h_{1} \in H . H=H^{+} \cup H$, in which $H^{-}$is the set of all negative hedges and $H^{+}$is the set of all positive ones of $X$. The possitive hedges increase semantic tendency and vise versa with negative hedges. Without loss of generality, it can be assumed that $H=\left\{h_{-1}<h_{-2}<\ldots<h_{-q}\right\}$ and $H^{+}=\left\{h_{1}<h_{2}<\ldots<h_{p}\right\}$.

If $X$ and $H$ are linearly ordered sets, then $A X=(X, G, C, H, \leq)$ is called linear hedge algebras, further more, if $A X$ is equipped with additional operations $\Sigma$ and $\Phi$ that are, respectively, infimum and supremum of $H(x)$, then it is called complete linear hedge algebras (ClinHA) and denoted $A X=(X, G, C, H, \Sigma, \Phi, \leq)$.

Fuzziness of vague terms and fuzziness measure are two concepts that are difficult to define in fuzzy set theory. However, HA can reasonably define these ones. Concretely, elements of $H(x)$ still express a certain meaning stemming from $x$, so we can interpret the set $H(x)$ as a model of the fuzziness of the term $x$. With regard to fuzziness measure, it can be formally defined by following difinitions.

Definition 2.1. Let $A X=(X, G, C, H, \leq)$ be a ClinHA. An $f m: X \rightarrow[0,1]$ is said to be a fuzziness measure of terms in $X$ if:

(1). $f m\left(c^{-}\right)+f m\left(c^{+}\right)=1$ and $\sum_{h \in H} f m(h u)=f m(u)$, for $\forall u \in X$; in this case $f m$ is called complete;

(2). For the constants $\boldsymbol{0}, \boldsymbol{W}$ and $\boldsymbol{1}, f m(\boldsymbol{0})=f m(\boldsymbol{W})=f m(\boldsymbol{1})=0$;

(3). For $\forall x, y \in X, \forall h \in H, \frac{f m(h x)}{f m(x)}=\frac{f m(h y)}{f m(y)}$, that is this proportion does not depend on specific elements and, hence, it is called fuzziness measure of the hedge $h$ and denoted by $\mu(h)$.

The condition (1) means that the primary terms and hedges under consideration are complete for modelling the semantics of the whole real interval of a physical variable. That is, except the primary terms and hedges under consideration, there are no more primary terms and hedges. (2) is intuitively evident. (3) seems also to be natural in the sense that applying a hedge $h$ to different vague concepts, the relative modification effect of $h$ is the same, i.e. this proportion does not depend on terms that they apply to.

The properties of fuzziness measure are determined clearly through the following proposition.

Proposition 2.1. For each fuzziness measure fm on $X$ the following statements hold:

(1). $f m(h x)=\mu(h) f m(x)$, for every $x \in X$;

(2). $f m\left(c^{-}\right)+f m\left(c^{+}\right)=1$;

(3). $\sum_{-q \leq i \leq p, i \neq 0} f m\left(h_{i} c\right)=f m(c), c \in\left\{c^{-}, c^{+}\right\}$;

(4). $\sum_{-q \leq i \leq p, i \neq 0} f m\left(h_{i} x\right)=f m(x)$;

(5). $\sum_{-q \leq i \leq-1} \mu\left(h_{i}\right)=\alpha$ and $\sum_{1 \leq i \leq p} \mu\left(h_{i}\right)=\beta$, where $\alpha, \beta>0$ and $\alpha+\beta=1$. 
HA build the method of quantifying the semantic of linguistic terms based on the fuzziness measures and hedges through $v$ mapping that fit to the conditions in the following definitions.

Definition 2.2. Let $A X=(X, G, C, H, \Sigma, \Phi, \leq)$ be a CLinHA. A mapping $v: X \rightarrow[0,1]$ is said to be an semantically quantifying mapping of $A X$, provided that the following conditions hold: (1). $v$ is a one-to-one mapping from $X$ into $[0,1]$ and preserves the order on $X$, i.e. for all $x, y \in X$, $x<y \Rightarrow v(x)<v(y)$ and $v(\mathbf{0})=0, v(\mathbf{1})=1$, where $\mathbf{0}, \mathbf{1} \in C$;

(2). $\forall x \in X, v(\Phi x)=$ infimum $v(H(x))$ and $v(\Sigma x)=$ supremum $v(H(x))$.

Semantically quantifying mapping $v$ is determined concretely as follows.

Definition 2.3. Let $f m$ be a fuzziness measure on $X$. A mapping $v: X \rightarrow[0,1]$, which is induced by $f m$ on $X$, is defined as follows:

(1). $v(\boldsymbol{W})=\theta=f m\left(c^{-}\right), v\left(c^{-}\right)=\theta-\alpha f m\left(c^{-}\right)=\beta f m\left(c^{-}\right), v\left(c^{+}\right)=\theta+\alpha f m\left(c^{+}\right)$;

(2). $v\left(h_{j} x\right)=v(x)+\operatorname{Sign}\left(h_{j} x\right)\left\{\sum_{i=\operatorname{Sign}(j)}^{j} f m\left(h_{i} x\right)-\omega\left(h_{j} x\right) f m\left(h_{j} x\right)\right\}$,

where $j \in\{j:-q \leq j \leq p \& j \neq 0\}=\left[-q^{\wedge} p\right]$

and $\omega\left(h_{j} x=\frac{1}{2}\left[1+\operatorname{Sign}\left(h_{j} x\right) \operatorname{Sign}\left(h_{p} h_{j} x\right)(\beta-\alpha)\right] \in\{\alpha, \beta\} ;\right.$

(3). $v\left(\Phi c^{-}\right)=0, v\left(\Sigma c^{-}\right)=\theta=v\left(\Phi c^{+}\right), v\left(\Sigma c^{+}\right)=1$, and for $j \in\left[-q^{\wedge} p\right]$,

$$
\begin{aligned}
v\left(\Phi h_{j} x\right)= & v(x)+\operatorname{Sign}\left(h_{j} x\right)\left\{\sum_{i=\operatorname{sign}(j)}^{j-s i g n(j)} \mu\left(h_{i}\right) f m(x)\right\}-\frac{1}{2}\left(1-\operatorname{Sign}\left(h_{j} x\right)\right) \mu\left(h_{j}\right) f m(x), \\
& v\left(\Sigma_{h_{j}} x\right)=\varphi(x)+\operatorname{Sign}\left(h_{j} x\right)\left\{\sum_{i=\operatorname{sign}(j)}^{j-\operatorname{sign}(j)} \mu\left(h_{i}\right) f m(x)\right\}+\frac{1}{2}\left(1+\operatorname{Sign}\left(h_{j} x\right)\right) \mu\left(h_{j}\right) f m(x) .
\end{aligned}
$$

The Sign function and fuzziness interval are determined in the following difinitions.

Definition 2.4. A function Sign: $X \rightarrow\{-1,0,1\}$ is a mapping which is defined recursively as follows, for $h, h^{\prime} \in H$ and $c \in\left\{c^{-}, c^{+}\right\}$:

(1). $\operatorname{Sign}(c)=-1, \operatorname{Sign}\left(c^{+}\right)=+1$;

(2). $\operatorname{Sign}(h c)=-\operatorname{Sign}(c)$, if $h$ is negative w.r.t. $c$; $\operatorname{Sign}(h c)=+\operatorname{Sign}(c)$, if $h$ is positive w.r.t. $c$;

(3). $\operatorname{Sign}\left(h^{\prime} h x\right)=-\operatorname{Sign}(h x)$, if $\quad h^{\prime} h x \neq h x$ and $h^{\prime}$ is negative w.r.t. $h$; $\operatorname{Sign}\left(h^{\prime} h x\right)=+$ $\operatorname{Sign}(h x)$, if $h^{\prime} h x \neq h x$ and $h^{\prime}$ is positive w.r.t. $h$.

(4). $\operatorname{Sign}\left(h^{\prime} h x\right)=0$ if $h^{\prime} h x=h x$.

Definition 2.5. The fuzziness interval of the linguistic terms $x \in X$, denoted by $\mathfrak{I}(x)$, is a subinterval of $[0,1]$, if $|\mathfrak{I}(x)|=f m(x)$ where $|\mathfrak{I}(x)|$ is the length of $\mathfrak{I}(x)$, and recursively determnied by the length of $x$ as follows:

(1). If length of $x$ is equal to $1(l(x)=1)$, that mean $x \in\left\{c^{-}, c^{+}\right\}$, then $\left|\mathfrak{I}\left(c^{-}\right)\right|=f m\left(c^{-}\right),|\Im(c+)|=$ $f m\left(c^{+}\right)$and $\mathfrak{I}\left(\boldsymbol{c}^{-}\right) \leq \mathfrak{J}\left(c^{+}\right)$; 
(2). Suppose that $n$ is the length of $x(l(x)=n)$ and fuzziness interval $\mathfrak{I}(x)$ has been definied with $|\mathfrak{I}(x)|=f m(x)$. The set $\left\{\mathfrak{I}\left(h_{j} x\right) \mid j \in\left[-q^{\wedge} p\right]\right\}$, where $\left[-q^{\wedge} p\right]=\{j \mid-q \leq j \leq-1$ or $1 \leq j \leq p\}$, is a partition of $\mathfrak{I}(x)$ and we have: for $\operatorname{Sgn}\left(h_{p} x\right)=-1, \mathfrak{I}\left(h_{p} x\right) \leq \mathfrak{I}\left(h_{p-1} x\right) \leq \ldots \leq \mathfrak{I}\left(h_{1} x\right) \leq \mathfrak{I}\left(h_{-1} x\right) \leq \ldots$ $\leq \mathfrak{I}\left(h_{-q} x\right) ;$ for $\operatorname{Sgn}\left(h_{p} x\right)=+1, \mathfrak{I}\left(h_{-q} x\right) \leq \mathfrak{I}\left(h_{-q+1} x\right) \leq \ldots \leq \mathfrak{I}\left(h_{-1} x\right) \leq \mathfrak{I}\left(h_{1} x\right) \leq \ldots \leq \mathfrak{I}\left(h_{p} x\right)$.

\section{THE PARTITIONING METHOD BASED ON HA}

Following fuzzy set approach, the linguistic terms used to qualitatively describe historical values of fuzzy time series, $X_{i}(t)(i=1,2, \ldots)$, are quantified by mean of fuzzy sets. In the HA approach, $X_{i}(t)$ are quantified by mean of the semantically quantifying mapping and fuzziness measure. So we need to adjust the definition of fuzzy time series for meeting with HA approach. This adjustment does not change the nature of fuzzy time series.

Definition 3.1. The definition of fuzzy time series based on HA

Let $X(t)(t=\ldots, 0,1,2, \ldots)$ a subset of $R^{1}$, be the universe of discourse of linguistic terms $X_{i}(t)(t=1,2, \ldots), F(t)$ is the collection of $X_{i}(t)$. Then $F(t)$ is called a fuzzy time series on $X(t)$.

The proposed method is expressed in the following:

Considering linguistic variable $l$, from domain of $l$ we can organize a hedge algebra $A X=$ $(X, G, H, \leq) . F(t)$ is the fuzzy time series containing a collection of linguistic terms of $l$, so $F(t) \subseteq X$ and the values of $F(t)$ are generated from $c^{-}$and $c^{+}$. The number of intervals on $U$ of $F(t)$ are equal to linguistic terms that are used to qualitatively describe historical values of $F(t)$. Each value of $F(t)$, a linguistic term, determines an interval which is the length of it's fuzziness interval. Formally, this method, called DI, comprises following steps:

Step 1: Determining the linguistic terms used to qualitatively describe the historical values of $F(t)$.

Step 2: Normalizing the linguistic terms so that they simultaneously generate from $c^{-}, c^{+}$and belong to HA $A X=(X, G, H$, $\leq)$. If we need to generate more linguistic term to match with the number of linguistic terms in Step 1, then if $H$ has more than two hedges, then we use two hedge $h_{g}, h_{e} \in H^{\prime}$ ( $H^{\prime}$ just contain $h_{g}$ and $h_{e}, H^{\prime} \neq H$ ) where $h_{g}$ is a nagative hedge, $h_{e}$ is a positive one and $f m\left(h_{g}\right)+f m\left(h_{e}\right)=1$. Next, choosing a linguistic term that has fuzziness interval containing the maximum amount of historical values, called $y$. From this one we can generate $h_{g} y$ and $h_{e} y$. Otherwise, if $H$ has only two hedges, then use these hedges to generate more hedges from $y$.

Step 3: Determining the number of intervals. These are equal to the number of linguistic terms in Step 2.

Step 4: Determining the size of intervals through determining fuzziness intervals of the linguistic terms by Proposition 2.1 .

The values of $F(t)$ may not simultaneously generate from certain generators, so Step 2 need to be performed. We can replace a linguistic term by a different linguistic term so that all of them belong to one HA.

Method DI is served as one step in the method of forecasting fuzzy time series. This method refers to the some ideas in [4] and [14]. We name this method FL.

Denoting $\operatorname{co} \mathfrak{I}(x)$ and $\operatorname{co} \vartheta(x)$, respectively, are fuzziness intervals and semantically quantifying values of $x$ that are mapped from $[0,1]$ to the universe of discourse, $U$, of $F(t)$. From 
here, when we mention "fuzziness interval" and "semantically quantifying value" of $x$ that means we are mentioning to $\operatorname{co} \mathfrak{I}(x)$ and $\operatorname{co} \vartheta(x)$.

Method FL, forecasting fuzzy time series:

Step 1: Applying DI to determine intervals on the universe of discourse of $F(t)$.

Step 2: Calculating the semantically quantifying values of linguistic terms that are used to qualitatively describe historical values of $F(t)$.

Step 3: Mining the fuzzy relationships among the linguistic terms.

To facilitate calculating, each linguistic terms, obtaining from Step 2, are denoted by $A i$ where $I=\overline{1, k}$. The fuzzy relationships are denoted: $A t \rightarrow A u(p) \ldots A v(q)$, where $A t, A u, \ldots A v$ are linguistic terms; $p, q$ are positive integers that refer to the number of iteration of $A u$, and $A v$ in the fuzzy relationships that have left side $A t$.

Step 4: Calculating forecasting values

Forecasting value of fuzzy time series at point $t+1$ is computed as follows:

Considering historical value of fuzzy time series at point $t$, denoted $f(t)$, if $f(t)$ belong to $\operatorname{cofm}(A t)$, then compute the forecasting value at point $t+1$ by following formula:

$$
\frac{p^{*} \operatorname{co\vartheta }\left(A u(h(A u))+\ldots+q^{*} \operatorname{co\vartheta }(A v(h(A v))\right.}{p+\ldots+q} \text { where } \operatorname{co\vartheta }(A i(h A i)) \text { is the semantically }
$$

quantifying value of $A i$ or $h A i$ which is chosen, $h$ is the negative or positive hedge mentioned in Step 2.

Let $\theta$ be average of values falling into $A i$ 's fuzziness interval, $\theta$ describes the density of historical values of $F(t)$ and tend to lean left, right or evenly distribute in this interval. $\operatorname{co} \vartheta(A i)$ or $\operatorname{co\vartheta }(h A i)$ are chosen depending on the distance from them to $\theta i$, dij where $j=1,2,3$. This distance is reflective of the suitability between semantics of linguistic term and distribution rule of historical values of fuzzy time series on intervals, so if any semantically quantifying value has minimum distance to $\theta i$, then that value will be chosen.

\section{RESULTS AND DISCUSSIONS}

In this section, method DI and FL are applied on regular time series used in some previous researchs. These time series are enrollments at University of Alabama, TAIEX index [15] and Unemployment rates [15]. From here, for short, these time series are briefly called Alabama, TAIEX and UEP. This paper takes the forecasting results of different methods used in paper [15] to compare with forecasting results of the proposed method.

Annually, it can use the linguistic terms such as [2,3] to qualitatively describe the enrollments at University of Alabama. However, we use the following linguistic terms to facilitate for applying the proposed method: very very low (A1), little very low (A2), very little low (A3), little little low (A4), little little hight (A5), very little hight (A6) and very hight (A7). These linguistic terms completely cover semantic description of the enrollments (from minimum enrollments to maximum enrollments). It can be seen that the linguistic terms belonging to domain of linguistic variable "enrollment" forming $\mathrm{HA}: A X=(X, G, H, \leq)$, where $G=\{$ low, hight $\}, H=\{$ very, little $\}, X=H(G)$.

Applying FL to forecast enrollments at University of Alabama as follows: 
Step 1: Applying DI to determine the intervals: Let $D_{\min }$ and $D_{\max }$, respectively, be minimum and maximum enrollment from 1971 to 1991 . Based upon $D_{\min }$ and $D_{\max }$ we define $U$ as $\left[D_{\min }-D_{1}\right.$, $\left.D_{\max }+D_{2}\right]$ where $D_{1}=55, D_{2}=663$, the same as [2-3], so $U=[13000,20000]$. The length of $U$, denote $L U, L U=20000-13000=7000$.

Table 1. The fuzzified historical enrollments.

\begin{tabular}{|c|c|c|c|c|c|}
\hline Year & $\begin{array}{c}\text { Actual } \\
\text { enrollment }\end{array}$ & $\begin{array}{l}\text { Fuzzified } \\
\text { enrollment }\end{array}$ & Year & $\begin{array}{c}\text { Actual } \\
\text { enrollment }\end{array}$ & $\begin{array}{l}\text { Fuzzifield } \\
\text { enrollment }\end{array}$ \\
\hline 1971 & 13055 & $A 1$ & 1982 & 15433 & $A 3$ \\
\hline 1972 & 13563 & $A 1$ & 1983 & 15497 & $A 3$ \\
\hline 1973 & 13867 & $A l$ & 1984 & 15145 & $A 3$ \\
\hline 1974 & 14696 & $A 2$ & 1985 & 15163 & $A 3$ \\
\hline 1975 & 15460 & $A 3$ & 1986 & 15984 & $A 4$ \\
\hline 1976 & 15311 & $A 3$ & 1987 & 16859 & $A 6$ \\
\hline 1977 & 15603 & $A 4$ & 1988 & 18150 & $A 7$ \\
\hline 1978 & 15861 & A4 & 1989 & 18970 & $A 7$ \\
\hline 1979 & 16807 & A6 & 1990 & 19328 & $A 7$ \\
\hline 1980 & 16919 & A6 & 1991 & 19337 & $A 7$ \\
\hline 1981 & 16388 & A5 & & & \\
\hline
\end{tabular}

The number of linguistic terms used to qualitatively describe the historical values of Alabama are 7, so $U$ is partitioned into 7 intervals. Specificially, the intervals are determined as follows:

Domain $U$ is mapped into $[0,1]$. If we suppose that 16000 is low, then it can set the parameters: $f m($ low $)=\frac{16000-13000}{20000-13000}=0.428$, so $f m($ hight $)=0.572$. Reversely mapping these values into $U$, we respectively have $\operatorname{co} \mathfrak{I}$ (low) and coI (hight): fm(low) x $L U=0.428 \times 7000=$ 2996, fm(hight) $\mathrm{x} L U=0.572 \times 7000=4004$.

It can choose: $\mu($ Little $)=0.4, \mu($ Very $)=0.6$. Based on these parameters we determined the fuzziness intervals of the linguistic terms that are also intervals on $U$ :

$\operatorname{co} \mathfrak{I}($ Al $)=\mu($ Very $) \times \mu($ Very $) \times \operatorname{co} \mathfrak{I}($ low $)=0.6 \times 0.6 \times 2996=1079$. The interval corresponding to $A 1$ is $[13000,14079)$. Similarly, we have the rest intervals: $[14079,14798)$, [14798, 15517), [15517, 15996), [15996, 16637), [16637, 17598), [17598, 20000).

Step 2: The semantically quantifying values of $A i$ and $h A i(i=1, \ldots, 7)$ are calculated by difinition 2.3 as follows:

$\operatorname{co\vartheta }(A 1)=\beta \times \cos ($ low $)-\cos (A 2)-\alpha \times \cos (A 1)=0.6 \times 2996-719-0.4 \times 1079=$ 13647. Similarly, we have semantically quantifying values of the rest linguistic terms. Based on historical values of Alabama, we computed $\theta i$ and $\operatorname{dij}(i=1, \ldots, 7, j=1,2,3)$. All of the values are shown in Table 2 in the following: 
Table 2. The values of $\operatorname{co\vartheta }(A i), \operatorname{co\vartheta }(h A i), \theta i$ and $d i j$.

\begin{tabular}{|c|c|c|c|}
\hline $\begin{array}{c}\operatorname{cov}(A 1)=13647 \\
d 11=169\end{array}$ & $\begin{array}{c}\operatorname{co\vartheta }(\text { VeryAl })=13388 \\
d 12=90\end{array}$ & $\begin{array}{c}\operatorname{co\vartheta }(\text { LittleAl })=13906 \\
d 13=428\end{array}$ & $\theta 1=13478$ \\
\hline $\operatorname{co\vartheta }(A 2)=14510$ & $\operatorname{co\vartheta }($ VeryA2 $)=14338$ & $\operatorname{co\vartheta }($ LittleA2 $)=14683$ & $\theta 2=14696$ \\
\hline$d 21=186$ & $d 22=358$ & $d 23=13$ & \\
\hline $\operatorname{co\vartheta }(A 3)=15229$ & $\operatorname{cov}($ VeryA3 $)=15056$ & $\operatorname{co\vartheta }($ LittleA3 $)=15402$ & $\theta 3=15335$ \\
\hline$d 31=106$ & $d 32=278$ & $d 33=67$ & \\
\hline $\operatorname{co\vartheta }(A 4)=15804$ & $\operatorname{cov}(\operatorname{VeryA} 4)=15689$ & $\operatorname{co\vartheta }($ LittleA4 $)=15919$ & $\theta 4=15816$ \\
\hline$d 41=12$ & $d 42=127$ & $d 43=103$ & \\
\hline $\operatorname{co\vartheta }(A 5)=16252$ & $\operatorname{co\vartheta }($ LittleA5 $)=16099$ & $\operatorname{co\vartheta }($ VeryA5 $)=16406$ & $\theta 5=16388$ \\
\hline$d 51=136$ & $d 52=289$ & $d 53=18$ & \\
\hline $\operatorname{co\vartheta }(A \sigma)=17021$ & $\operatorname{co\vartheta }($ LittleA6 $)=16790$ & $\operatorname{co\vartheta }($ VeryA6 $)=17252$ & $\theta 6=16862$ \\
\hline$d 61=159$ & $d 62=71$ & $d 63=390$ & \\
\hline $\operatorname{co\vartheta }(A 7)=18559$ & $\operatorname{co\vartheta }($ LittleA7 $)=17982$ & $\operatorname{co\vartheta }($ VeryA7 $)=19135$ & $\theta 7=18932$ \\
\hline$d 71=374$ & $d 72=950$ & $d 73=203$ & \\
\hline
\end{tabular}

In Table 2, the grey cells have $\operatorname{co\vartheta }(A i)$ or $\operatorname{co\vartheta }(h A i)$ which is chosen.

Step 3: Based on Table 1 we mined the fuzzy relationships as follows:

Table 3. Group of fuzzy relationships.

\begin{tabular}{ll}
\hline Group 1 & $A 1 \rightarrow A 1(2), A l \rightarrow A 2$ \\
Group 2 & $A 2 \rightarrow A 3$ \\
Group 3 & $A 3 \rightarrow A 3(4), A 3 \rightarrow A 4(2)$ \\
Group 4 & $A 4 \rightarrow A 4, A 4 \rightarrow A 6(2)$ \\
Group 5 & $A 5 \rightarrow A 3$ \\
Group 6 & $A 6 \rightarrow A 5 A 6 A 7$ \\
Group 7 & $A 7 \rightarrow A 7(4)$ \\
\hline
\end{tabular}

Step 4: Based on the data from Table 1 and Table 3, the forecasting values of the years from 1972 to 1992 are calculated by method of FL as follows:

[1972]: The linguistic term used to qualitatively describe the historical value of 1971 is $A 1$ and from Table 3 we can see that the fuzzy relationships have left side $A l$ belonging to Group 1: $A 1 \rightarrow A 1, A 1 \rightarrow A 2$. The picked semantically quantifying values correspond to $A 1$ and $A 2$ respectively are $\operatorname{cov}($ VeryA1 $)=13388, \operatorname{cov}($ LittleA2 $)=14683$. So the forecasting value of 1972 is $\frac{1}{3} \times(13388 \times 2+14683)=13820$. Similar to that, we have the forecasting values of the rest 
years. The forecasting result is shown as well as different forecasting results (belonging to some recently other methods) in the following Table 4.

Table 4. Comparing forecasting result on Alabama.

\begin{tabular}{lcccccc}
\hline Year & Actual value & Wang 2013 & Wang 2014 & Chen 2013 & Lu 2015 & $\begin{array}{c}\text { Proposed } \\
\text { method }\end{array}$ \\
\hline 1972 & 13563 & 13486 & 13944 & 14347 & 14279 & 13820 \\
1973 & 13867 & 14156 & 13944 & 14347 & 14279 & 13820 \\
1974 & 14696 & 15215 & 13944 & 14347 & 14279 & 13820 \\
1975 & 15460 & 15906 & 15328 & 15550 & 15392 & 15402 \\
1976 & 15311 & 15906 & 15753 & 15550 & 15392 & 15536 \\
1977 & 15603 & 15906 & 15753 & 15550 & 15392 & 15536 \\
1978 & 15861 & 15906 & 15753 & 15550 & 16467 & 16461 \\
1979 & 16807 & 16559 & 16279 & 16290 & 16467 & 16461 \\
1980 & 16919 & 16559 & 17270 & 17169 & 17161 & 17444 \\
1981 & 16388 & 16559 & 17270 & 17169 & 17161 & 17444 \\
1982 & 15433 & 16559 & 16279 & 16209 & 14916 & 15402 \\
1983 & 15497 & 15906 & 15753 & 15550 & 15392 & 15536 \\
1984 & 15145 & 15906 & 15753 & 15550 & 15392 & 15536 \\
1985 & 15163 & 15906 & 15753 & 15550 & 15392 & 15536 \\
1986 & 15984 & 15906 & 15753 & 15550 & 15470 & 15536 \\
1987 & 16859 & 16559 & 16279 & 16290 & 16467 & 16461 \\
1988 & 18150 & 16559 & 17270 & 17169 & 17161 & 17444 \\
1989 & 18970 & 19451 & 19466 & 18907 & 19257 & 19135 \\
1990 & 19328 & 18808 & 18933 & 18907 & 19257 & 19135 \\
1991 & 19337 & 18808 & 18933 & 18907 & 19257 & 19135 \\
1992 & 18876 & 18808 & 18933 & 18907 & 19257 & 19135 \\
& RMSE & 578.3 & 506.0 & 486.3 & 445.2 & 441.3 \\
\hline & & & & & & \\
\hline
\end{tabular}

The root mean square error (RMSE) criteria is usually used to estimate forecasting perfomance in the literature: RMSE $=\sqrt{\frac{1}{n} \sum_{i=1}^{n}\left(x^{\prime}{ }_{i}-x_{i}\right)^{2}}$, where $x_{i}{ }^{\prime}$ is the forecasting value, $x_{i}$ is historical value and $n$ is the number of forecasting values. Applying RMSE for the forecasting result of the proposed method we have: $\mathrm{RMSE}=441.3$.

Similarly, applying FL for TAIEX 1992 [15] with 7 intervals we have Table 5 in the following: 
Table 5. Comparing forecasting result on TAIEX.

\begin{tabular}{|c|c|c|c|c|c|c|}
\hline Date & Actual data & Wang 2013 & $\begin{array}{l}\text { Chen } \\
2013\end{array}$ & $\begin{array}{l}\text { Wang } \\
2014\end{array}$ & Lu 2015 & $\begin{array}{c}\text { Proposed } \\
\text { method }\end{array}$ \\
\hline 02/12/1992 & 3635.7 & 3629.3 & 3740.9 & 3564.5 & 3693.1 & 3709.8 \\
\hline 03/12/1992 & 3614.1 & 3629.3 & 3740.9 & 3564.5 & 3693.1 & 3709.8 \\
\hline 04/12/1992 & 3651.4 & 3629.3 & 3740.9 & 3564.5 & 3693.1 & 3709.8 \\
\hline 05/12/1992 & 3727.9 & 3629.3 & 3740.9 & 3564.5 & 3693.1 & 3709.8 \\
\hline 07/12/1992 & 3755.8 & 3629.3 & 3740.9 & 3859.9 & 3693.1 & 3709.8 \\
\hline 08/12/1992 & 3761 & 3629.3 & 3740.9 & 3859.9 & 3693.1 & 3709.8 \\
\hline 09/12/1992 & 3776.6 & 3629.3 & 3740.9 & 3859.9 & 3693.1 & 3709.8 \\
\hline $10 / 12 / 1992$ & 3746.8 & 3629.3 & 3740.9 & 3859.9 & 3693.1 & 3709.8 \\
\hline 11/12/1992 & 3734.3 & 3629.3 & 3740.9 & 3859.9 & 3693.1 & 3709.8 \\
\hline $12 / 12 / 1992$ & 3742.6 & 3629.3 & 3740.9 & 3859.9 & 3693.1 & 3709.8 \\
\hline $14 / 12 / 1992$ & 3696.8 & 3629.3 & 3740.9 & 3859.9 & 3693.1 & 3709.8 \\
\hline $15 / 12 / 1992$ & 3688.3 & 3629.3 & 3740.9 & 3564.5 & 3693.1 & 3709.8 \\
\hline $16 / 12 / 1992$ & 3674.9 & 3629.3 & 3740.9 & 3564.5 & 3693.1 & 3709.8 \\
\hline $17 / 12 / 1992$ & 3668.7 & 3629.3 & 3740.9 & 3564.5 & 3693.1 & 3709.8 \\
\hline $18 / 12 / 1992$ & 3658 & 3629.3 & 3740.9 & 3564.5 & 3693.1 & 3709.8 \\
\hline 21/12/1992 & 3576.1 & 3629.3 & 3740.9 & 3564.5 & 3693.1 & 3709.8 \\
\hline $22 / 12 / 1992$ & 3578 & 3629.3 & 3477.1 & 3564.5 & 3519.4 & 3442.3 \\
\hline 23/12/1992 & 3448.2 & 3629.3 & 3477.1 & 3564.5 & 3519.4 & 3442.3 \\
\hline 24/12/1992 & 3456 & 3629.3 & 3477.1 & 3413.3 & 3519.4 & 3442.3 \\
\hline 28/12/1992 & 3327.7 & 3629.3 & 3477.1 & 3413.3 & 3519.4 & 3442.3 \\
\hline 29/12/1992 & 3377.1 & 3629.3 & 3368.1 & 3413.3 & 3519.4 & 3491.4 \\
\hline \multicolumn{2}{|c|}{ RMSE } & 114.2 & 85.7 & 107.2 & 75.7 & 68.9 \\
\hline
\end{tabular}


Also Applying FL for UNE [15] with 9 intervals, the forecasting result is presented in the following Table 6:

Table 6. Comparing forecasting result on UNE.

\begin{tabular}{ccccccc}
\hline Date & Actual data & Wang 2013 & $\begin{array}{c}\text { Chen } \\
2013\end{array}$ & Wang 2014 & Lu 2015 & $\begin{array}{c}\text { The proposed } \\
\text { method }\end{array}$ \\
\hline $02 / 01 / 2013$ & 7.7 & 7.39 & 7.60 & 7.62 & 7.58 & 7.51 \\
$03 / 01 / 2013$ & 7.5 & 7.39 & 7.60 & 7.62 & 7.58 & 7.51 \\
$04 / 01 / 2013$ & 7.5 & 7.39 & 7.60 & 7.62 & 7.58 & 7.51 \\
$05 / 01 / 2013$ & 7.5 & 7.39 & 7.60 & 7.62 & 7.58 & 7.51 \\
$06 / 01 / 2013$ & 7.5 & 7.39 & 7.60 & 7.62 & 7.58 & 7.51 \\
$07 / 01 / 2013$ & 7.3 & 7.39 & 7.60 & 7.62 & 7.58 & 7.51 \\
$08 / 01 / 2013$ & 7.2 & 7.39 & 7.12 & 7.13 & 7.07 & 6.99 \\
$09 / 01 / 2013$ & 7.2 & 6.89 & 7.12 & 7.13 & 7.07 & 6.99 \\
$10 / 01 / 2013$ & 7.2 & 6.89 & 7.12 & 7.13 & 7.07 & 6.99 \\
$11 / 01 / 2013$ & 7.0 & 6.89 & 7.12 & 7.13 & 7.07 & 6.99 \\
$12 / 01 / 2013$ & 6.7 & 6.89 & 7.12 & 7.13 & 7.07 & 6.99 \\
RMSE & & 0.20 & 0.18 & 0.19 & 0.17 & 0.16 \\
\hline
\end{tabular}

Comparing forecasting results of the proposed method with some forecasting result of recently different methods on regular time series such as Alabama, TAIEX, UNE in Table 4, Table 5 and Table 6 show that the proposed method gives better forecasting performance. Besides, the proposed method only use arithmetic operations with simple way to calculate forecasting result.

\section{CONCLUSION}

This paper presented a novel method of partitioning the universe of discourse, and used this method in the method of using fuzzy time series to forecast time series, to improve forecasting performance. The proposed method is formed by mean of the linguistic terms that are used to qualitatively describe the historical values of fuzzy time series. Based on the linguistic terms, the number of intervals, corresponding to the number of linguistic terms, and length of intervals, corresponding to the fuzziness intervals, are determined. 
From the experimental results on the regular time series, compare to forecasting result of different methods, we can see that when using the proposed method to model fuzzy time series gives better forecasting accuracy. The proposed method also shows that it is rather simple because of using only arithmetic operations and simple way to calculate forecasting values.

\section{REFERENCES}

1. $\quad$ Song Q., Chissom B.S - Fuzzy time series and its models, Fuzzy Sets and Systems 54 (3) (1993a) 269-277.

2. Song Q., Chissom B.S - Forecasting enrollments with fuzzy time series - Part I, Fuzzy Sets and Systems 54 (1) (1993b) 1-9.

3. Song Q., Chissom B. S. - Forecasting enrollments with fuzzy time series, Part II, Fuzzy Sets and Systems 62 (1) (1994) 1-8.

4. Shyi-Ming Chen - Forecasting enrollments based on fuzzy time series, Fuzzy Sets and Systems 81 (1996) 311-319.

5. Kunhuang Huarng - Efective lengths of intervals to improve forecasting in fuzzy time series, Fuzzy Sets and Systems 123 (2001) 387-394

6. Kunhuang Huarng - Heuristic models of fuzzy time series for forecasting, Fuzzy Sets and Systems 123 (2001) 369-386.

7. Shyi-Ming Chen, Nien-Yi Chung - Forecasting enrollments using high-order fuzzy time series and genetic algorithms, International journal of intelligent systems 21 (2006) 485501.

8. Tahseen Ahmed Jilani, Syed Muhammad Aqil Burney, and Cemal Ardil - Fuzzy metric approach for fuzzy time series forecasting based on frequency density based partitioning, International Journal of Computer, Information, Systems and Control Engineering 4 (7)(2010) 39-44.

9. Shyi-Ming Chen, Chia-Ching Hsu - A new method to forecast enrollments using fuzzy time Series, International Journal of Applied Science and Engineering 2 (3) (2004) 234244.

10. Kunhuang Huarng, Tiffany Hui-Kuang Yu - Ratio-based lengths of intervals to improve fuzzy time series forecasting, IEEE transactions on systems, man, and cybernetics-part b: cybernetics 36 (2) (2006) 328-340.

11. Shyi-Ming Chen, Pei-Yuan Kao - TAIEX forecasting based on fuzzy time series, particle swarm optimization techniques and support vector machines, Information Sciences 247 (2013) 62-71.

12. Lizhu Wang, Xiaodong Liu, Witold Pedrycz - Effective intervals determined by information granules to improve forecasting in fuzzy time series. Expert Systems with Applications 40 (2013) 5673-5679.

13. Lizhu Wang, Xiaodong Liu, Witold Pedrycz, Yongyun Shao - Determination of temporal information granules to improve forecasting in fuzzy time series, Expert Systems with Applications 41 (2014) 3134-3142.

14. Eren Bas, Vedide Rezan Uslu, Ufuk Yolcu, Erol Egrioglu - A modified genetic algorithm for forecasting fuzzy time series, Applied Intelligence 41 (2014) 453-463. 
15. Wei Lu, XueyanChen, WitoldPedrycz, XiaodongLiua, JianhuaYang - Using interval information granules to improve forecasting in fuzzy time series, International Journal of Approximate Reasoning 57 (2015) 1-18.

16. Nguyen Cat Ho, Nguyen Van Long - Fuzziness measure on complete hedge algebras and quantifying semantics of terms in linear hedge algebras, Fuzzy Sets and Systems 158 (2007) $452-471$.

17. Cat Ho Nguyen, Witold Pedrycz, Thang Long Duong, Thai Son Tran - A genetic design of linguistic terms for fuzzy rule based classifiers, International Journal of Approximate Reasoning 54 (2013) 1-21. 